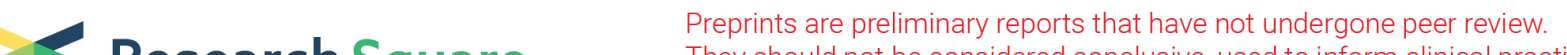 Research Square They should not be considered conclusive, used to inform clinical practice, or referenced by the media as validated information.
}

\section{Timely and Appropriate Administration of Inhaled Argon Provides Better Outcomes for tMCAO Mice: a Controlled, Randomized and Double-blind Animal Study}

\section{Juan He}

Nantong University Affiliated Hospital: Affiliated Hospital of Nantong University

Ke Xue

Nantong University Institute of Special Environmental Medicine

Jiayi Liu

Nantong University Institute of Special Environmental Medicine

Jin-hua Gu

Nantong University

\section{Bin Peng}

Nantong University Institute of Special Environmental Medicine

Lihua Xu

Nantong University Institute of Special Environmental Medicine

\section{Guohua Wang}

Nantong University Institute of Special Environmental Medicine

\section{Zhenglin Jiang}

Nantong University Institute of Special Environmental Medicine

Xia Li ( $D$ lixia7979@ntu.edu.cn )

Nantong University Institute of Special Environmental Medicine https://orcid.org/0000-0003-08046567

\section{Yunfeng Zhang}

Nantong University Affiliated Hospital: Affiliated Hospital of Nantong University

\section{Research}

Keywords: argon, inert gases, neuroprotective agents, transient middle cerebral artery occlusion, doubleblind study

Posted Date: August 23rd, 2021

DOI: https://doi.org/10.21203/rs.3.rs-826456/v1 
License: (c) (i) This work is licensed under a Creative Commons Attribution 4.0 International License. Read Full License

Version of Record: A version of this preprint was published at Neurocritical Care on February 8th, 2022. See the published version at https://doi.org/10.1007/s12028-022-01448-9. 


\section{Abstract}

Background: Inhaled argon (iAr) has shown promising therapeutic efficacy for acute ischemic stroke (AIS) and exhibited impressive advantages over other inert gases as a neuroprotective agent. However, the optimal dose, duration and time point of iAr for AIS are unknown. Here, we explored variable iAr schedules and evaluated the neuroprotective effects of acute iAr administration on lesion volume, brain edema, and neurological function in a mouse model of cerebral ischemic/reperfusion (I/R) injury.

Methods: Adult ICR mice were randomly subjected to sham, moderate $(1.5 \mathrm{~h})$ or severe $(3 \mathrm{~h})$ transient middle cerebral artery occlusion ( $\mathrm{tMCAO}$ ). One hour after $\mathrm{IMCAO}$, the mice were randomized to variable iAr protocols or air (iCtr). General and focal deficit scores were assessed during double-blind treatment. Infarct volume, overall recovery and brain edema were analyzed $24 \mathrm{~h}$ after cerebral I/R injury.

Results: Compared with those in the tMCAO only group, lesion volume $(\mathrm{p}<0.001)$ and neurologic outcome (general, $p<0.001$; focal, $p<0.001$ ) were significantly improved in the iAr group, which was assigned to argon inhalation $1 \mathrm{~h}$ after ischemia (before the onset of reperfusion). Short-term argon treatment ( $1 \mathrm{~h}$ or 3 h) showed significantly better outcomes with regard to infarct volume $(p<0.01)$ compared to argon inhalation for $24 \mathrm{~h}$. The concentration of argon inhalation was confirmed to be a key factor in improving the focal neurological outcome relative to that in the IMCAO group, and higher concentrations showed better effects. In addition, even though ischemia research has shown an increase in cerebral damage proportional to ischemia time, argon administration showed significant neuroprotective efficacy on infarct volume $(p<0.001)$, neurological deficits (general, $p<0.001$; focal, $p<0.001)$, weight recovery $(p<0.001)$, and edema $(p<0.001)$ in general, particularly in moderate stroke.

Conclusions: Timely argon inhalation before the onset of reperfusion showed optimal neurological outcomes and minimal infarct volumes. Moreover, an appropriate duration of argon administration was important for better neuroprotective efficacy. These findings may provide vital guidance for using argon as a neuroprotective agent and moving to clinical trials in acute ischemic stroke.

\section{Introduction}

Acute ischemic stroke (AIS) is a major global disease characterized by a high incidence, disability rate and mortality rate and is one of the main reasons for admission to the neurological intensive care unit[1, 2]. In recent decades, neuroprotective agents have shown efficacy and safety in experimental animal models of AIS. However, most potential candidate neuroprotective drugs have failed in preclinical and clinical trials[3]. In recent years, medical gases, such as xenon, helium, argon, volatile anesthetics (sevoflurane, isoflurane), $\mathrm{H}_{2} \mathrm{~S}, \mathrm{H}_{2}$, etc., used as neuroprotective agents, which are alternative or adjuvant methods for thrombolysis and endovascular therapy during multiple phases of AIS, have gained considerable attention[4-7]. Unlike other therapeutics, medical gases can easily diffuse into target tissue, cross the blood-brain barrier (BBB), and reach all parts of the brain, with the advantages of rapid onset and offset and titratability. Recently, inhaled argon (iAr) has become a promising choice due to 
encouraging neuroprotective effects demonstrated in multiple species and a range of experimental models of stroke, hypoxic-ischemic encephalopathy (HIE) and trauma[8-23], with the unique advantages of being readily available, easy to administer and transport, lacking anesthetic properties under normal pressure and not inhibiting the thrombolytic efficacy of tissue plasminogen activators at high concentrations (75\%)[24-29].

Despite these promising data for argon neuroprotection in various animal models of diseases, several studies have suggested that iAr results in insignificant improvement or even detrimental outcomes under certain prognostic indices or circumstances $[11,15,16,30]$, such as the timing, duration and optimal dose of applications. In the transient middle cerebral artery occlusion (tMCAO) model, for example, David and colleagues [11]showed that argon at 50 vol\% administered for $1 \mathrm{~h}$ during reperfusion resulted in an increase in subcortical brain damage but improved infarct volume followed by argon at 50 vol\% for $3 \mathrm{~h}$ during ischemia, as found by Ryang and colleagues[10]. Another paper by Ryang et al[31] showed that $50 \%$ argon $/ 50 \%$ oxygen inhaled for $3 \mathrm{~h}$ during ischemia resulted in elevated expression of not only several neuroprotective growth factors (TGF $\beta, N G F$, and VEGF) but also several inflammatory cytokines (IL-1 $\beta$, IL-6, and iNOS). Similarly, Ma and colleagues[16] found that argon failed to improve infarct size in a recent study in which rats were exposed to $24 \mathrm{~h}$ inhalation of $70 \%$ argon/30\% oxygen during reperfusion. Thus, the systematic scheme of argon treatment is a key factor required for its therapeutic efficacy. However, few studies have been performed on systematic experimental regimens of argon administration for neuroprotection, especially in tMCAO models.

To explore the optimal treatment regimen selection of argon inhalation, we investigated the therapeutic efficacy of argon administration in IMCAO-induced severe ischemic stroke at different initiation points, durations and concentrations of argon by analyzing infarct volume, deficit scores and overall recovery. We also used brain edema to assess whether argon influences AIS complications in two different stroke models induced by different ischemic durations, mimicking moderate or severe AIS.

\section{Materials And Methods}

\section{Animals}

All experiments and methods were carried out in accordance with the relevant guidelines and regulations. All animal procedures were approved by the Institutional Animal Care and Use Committee of Nantong University. Male ICR mice weighing 25-30 g were obtained from the Experimental Animal Center of Nantong University and housed in a vivarium with a 12-h light/dark cycle and free access to food and water. The body temperature of each animal was maintained at $37 \pm 0.5^{\circ} \mathrm{C}$ throughout the procedure using a heat pad.

\section{Mouse model of transient MCAO}

Mice were anesthetized with isoflurane. Transient MCAO was performed as described previously[32]. A midline ventral cervical skin incision was made to expose the right common carotid artery (CCA), external 
carotid artery (ECA), and internal carotid artery (ICA). After ligation at the proximal end of the common carotid artery, a 6-0 monofilament with a silicon-coated tip (6023PK5Re, Doccol Corporation) was inserted and positioned at the origin of the MCA to occlude it. The same surgical procedure was performed in sham-operated animals except for occlusion of the artery. The signs of a successful surgery included the following: flexion or reduced grasping ability of the left foreleg and spontaneous circling or toppling to the left. Cerebral blood flow was monitored throughout the surgery using laser Doppler flowmetry. At the time of reperfusion, which varied depending on the experiment, rats were anaesthetized, the neck wound was reopened, and the suture was removed. Body temperature was maintained between 36.5 and $37.5^{\circ} \mathrm{C}$ during the procedure. After $24 \mathrm{~h}$ of post-MCAO reperfusion, the mice were euthanized for the following experiments.

\section{Gas exposure system and experimental protocols}

After the time of recovery, mice were exposed to $79 \% \mathrm{i} \operatorname{Ar}(79 \%$ argon in $21 \%$ oxygen) or $39 \% \mathrm{i} \operatorname{Ar}(39 \%$ argon/40\% nitrogen/21\% oxygen) for the specified time in a custom-built gas delivery system consisting of a gas intervention box with a total volume of approximately $3.5 \mathrm{~L}$ connected to a mixed gas bottle that can display the flow rate. The system was flushed with more than four times the system volume of the mixed gases before the gas transmission circulation system was established.

Protocol 1: The mice were divided into four groups: sham, tMCAO, iAr treatment $1 \mathrm{~h}$ after stroke onset, and iAr treatment $3 \mathrm{~h}$ after stroke onset. All mice in each treatment group were administered $79 \%$ argon in $21 \%$ oxygen ventilation of iAr for $3 \mathrm{~h}$. Two different neurological deficit scores were obtained after tMCAO with reperfusion, the 3-hr iAr application, and $1 \mathrm{~d}$ of recovery. Mice were sacrificed $24 \mathrm{~h}$ after reperfusion, and coronal brain slices stained with TTC were saved for pathological evaluation.

Protocol 2: Based on the result of Protocol 1, inhaled $79 \%$ argon was used $1 \mathrm{~h}$ after stroke onset to provide neuroprotection in this experiment. For this time-dependent study, the treatment group received $79 \% \mathrm{i} \operatorname{Ar}(79 \%$ argon in $21 \%$ oxygen) for $1 \mathrm{~h}, 3 \mathrm{~h}$, or $24 \mathrm{~h}$ at $1 \mathrm{~h}$ after stroke onset. The mice were divided into five groups: sham, tMCAO, 79\% iAr for $1 \mathrm{~h}, 79 \%$ iAr for $3 \mathrm{~h}$, and $79 \%$ iAr for $24 \mathrm{~h}$. Measurements of the focal deficits to obtain neurological deficit scores were performed $24 \mathrm{~h}$ after reperfusion. After evaluation of neurological deficits, mice were sacrificed for histological assessment performed by staining brain areas.

Protocol 3: In this dose-dependent study, each treatment group received either $39 \%$ or $79 \% \mathrm{iAr} 1 \mathrm{~h}$ after stroke onset. The mice were divided into four groups: sham, tMCAO, 39\% iAr for $3 \mathrm{~h}$, and $79 \%$ iAr for $3 \mathrm{~h}$. Measured of the focal deficits to obtain neurological deficit scores were performed $24 \mathrm{~h}$ after reperfusion. Mice were sacrificed $24 \mathrm{~h}$ after reperfusion, and coronal brain slices stained with TTC were saved for pathological evaluation.

Protocol 4: Mice were divided into sham, tMCAO, and 79\% iAr for $3 \mathrm{~h}$ groups in two different ischemia duration models ( $1.5 \mathrm{~h} / 24 \mathrm{~h} ; 3 \mathrm{~h} / 24 \mathrm{~h})$, inducing moderate and severe ischemic stroke. Mice were subjected to $1.5 \mathrm{~h} / 3 \mathrm{~h}$ MCAO followed by reperfusion, and both groups were exposed to $79 \% \mathrm{i} \operatorname{Ar}(79 \%$ 
argon/21\% oxygen) $1 \mathrm{~h}$ after stroke onset for $3 \mathrm{~h}$. Twenty-four hours after reperfusion, two different neurological deficit scores were evaluated, and mice were then sacrificed for measurement of the infarct volume.

Protocol 5: The argon treatment approach was performed, and neurological deficit scores were evaluated as in Protocol 4. Mice were sacrificed at $24 \mathrm{~h}$ for determination of brain water content.

\section{Mortality rates and evaluation of neurological deficits}

Mortality rates were calculated $24 \mathrm{~h}$ after MCAO as a parameter of this study. Mice that died during the reperfusion stage within $24 \mathrm{~h}$ were excluded from other outcome parameter analyses. The neurologic assessment was conducted by a researcher who was blinded to the experimental groups. The neurological behavior of the mice was scored $24 \mathrm{~h}$ after MCAO/R according to Clark's scoring system[33] using a general neurological scale (0-28) and a focal neurological scale (0-28). Six areas were assessed for the general score, and seven areas were assessed for the focal score to evaluate the severity of neurological deficits after ischemic stroke. The focal neurological scale was based on the following seven tests, each of which was scored as 0-4 points: (1) body symmetry, (2) gait, (3) climbing, (4) circling behavior, (5) forelimb symmetry, (6) compulsory circling, and (7) whisker response.

\section{Measurement of cerebral infarct volume}

After neurological evaluation, the mice were euthanized, and brains were collected for measurement of the infarct volume. The brain was removed rapidly and placed at $-20^{\circ} \mathrm{C}$ for $20 \mathrm{~min}$. Coronal sections were cut into five 2-mm-thick slices, and the slices were immersed in 1\% 2,3,5-tripenyltetrazolium chloride (TTC) in phosphate-buffered saline at $37^{\circ} \mathrm{C}$ for $20 \mathrm{~min}$ followed by overnight immersion in $4 \%$ paraformaldehyde. The infarct area of each slice was demarcated and analyzed using ImageJ by an investigator blinded to the experimental groups. Infarct volume was calculated by integration of the infarct areas for all slices from each brain, and each section was measured by a blinded researcher. The infarct area was determined by subtracting the area of noninfarcted tissue in the ipsilateral hemisphere from that of the intact contralateral hemisphere to correct for brain swelling[34].

\section{Quantification of brain water content}

After neurological measurement, we used the wet-dry method to assess the brain water content[35]. Mice were euthanized under deep anesthesia. Each hemisphere was weighed (wet weight) and left in a desiccating oven at $95^{\circ} \mathrm{C}$ overnight, and then the dried hemispheres were weighed again (dry weight). The percentage of brain water content of each part was calculated as [(wet weight-dry weight)/wet weight] $\times 100 \%$.

\section{Statistical analysis}

Statistical analysis was performed using GraphPad Prism software (GraphPad Prism 8.0.1., GraphPad software, USA). Data are expressed as the mean \pm standard error (mean \pm SEM). One-way analysis of 
variance (ANOVA) was used to evaluate the differences among multiple groups if the data exhibited a normal distribution and homogeneity of variance. Two-way analysis of ANOVA was used to measure the effects of 2 factors simultaneously, with post hoc Bonferroni correction for multivariate analyses. Otherwise, the differences were assessed by a nonparametric test. A P value less than 0.05 was considered indicative of statistical significance.

\section{Results}

\section{iAr administration initiated during early ischemia-reperfusion attenuated brain injury better than administration after reperfusion}

To assess whether argon treatment at different initiation points has neuroprotective effects after severe ischemic stroke induced by delayed reperfusion (I/R; $3 \mathrm{~h} / 24 \mathrm{~h}$ ) and to explore the best time point for iAr administration, we applied two different treatment strategies that are closer to the actual clinical situation (iAr administered at $1 \mathrm{~h}$ after stroke onset; iAr administered at $3 \mathrm{~h}$ after stroke onset). We analyzed the brain infarct volume, neurological deficit scores and weight recovery $24 \mathrm{~h}$ after reperfusion to assess the severe ischemic stroke outcome. The mice were reperfused $3 \mathrm{~h}$ after MCAO, inducing severe ischemic stroke. We found that in the total infarct volume analysis, the group that inhaled argon $1 \mathrm{~h}$ after stroke onset (during ischemia) showed significantly lower volumes $24 \mathrm{~h}$ after reperfusion than the $\mathrm{MCAO}$ alone group, and a significant difference was observed between the two argon treatment groups. (Fig. 2B; $\mathrm{P}<$ 0.001). Consistent with the reduced infarct volume, the two different neurological deficit scores were also significantly improved in the group receiving iAr $1 \mathrm{~h}$ after stroke onset (Fig. $2 \mathrm{C}-\mathrm{D} ; \mathrm{P}<0.001$ ). Overall recovery, based on weight, was also improved in the group receiving iAr $1 \mathrm{~h}$ after stroke onset (Fig. $2 \mathrm{E}$; $\mathrm{P}<$ 0.01). The group receiving iAr $3 \mathrm{~h}$ after stroke onset exhibited improved neurological deficit scores compared with the IMCAO alone group assessed by the focal deficits but not the general deficits (Fig. 2D; focal, $P<0.05)$. In this group, however, infarct volume was not reduced, nor was weight recovery improved (Fig. 2B, E; $P>0.05$ ). Inhaled argon administration at the two initial intervention time points showed a trend toward decreased mortality in mice compared with $\mathrm{tMCAO}$ alone. However, the trend did not reach statistical significance (Fig. 2F). These results suggested that iAr administration $1 \mathrm{~h}$ after stroke onset (during ischemia) improved ischemic brain injury, attenuated the general deficit scores and improved weight recovery in severe ischemic stroke but not in animals administered iAr $3 \mathrm{~h}$ after stroke onset (during reperfusion).

\section{Inhaled argon administration attenuated brain injury in a time-dependent manner}

To further evaluate the relationship between the inhalation duration of iAr during ischemia and its neuroprotective effect on $\mathrm{TMCAO}$-induced severe ischemic stroke in mice, we tested the neuroprotective effects of three different durations of iAr (argon application for $1 \mathrm{~h}, 3 \mathrm{~h}$, or $24 \mathrm{~h}$ ) given $1 \mathrm{~h}$ after stroke onset. At $1 \mathrm{~d}$ after MCAO, we sectioned and stained brains with TTC, a substrate for mitochondrial respiration (Fig. 3A). We estimated infarct volumes by assessing the staining of brain areas (Fig. 3A-B). The infarct volume in the brain slices stained with TTC was the lowest in the group receiving $1 \mathrm{~h}$ argon 
administration, followed by the $3 \mathrm{~h}$ argon administration, $24 \mathrm{~h}$ argon administration and control groups, which is indicative of the neuroprotective effect of iAr, with 1-3 $\mathrm{h}$ of iAr providing the most substantial effect (Fig. 3B). Argon inhalation for both $1 \mathrm{~h}$ and $3 \mathrm{~h}$ showed significantly lower volumes than inhalation for $24 \mathrm{~h}(1 \mathrm{~h}$ treatment group vs. $24 \mathrm{~h}$ treatment group, $\mathrm{P}<0.01 ; 3 \mathrm{~h}$ treatment group vs. $24 \mathrm{~h}$ treatment group, $P<0.01$ ), but no significant difference was observed between the $1 \mathrm{~h}$ and $3 \mathrm{~h}$ treatment groups (Fig. 3B, $\mathrm{P}>0.05$ ). We also noted that iAr improved adverse neurologic outcomes (neuroscore) only when it was inhaled for $3 \mathrm{~h}$; when iAr was administered for $1 \mathrm{~h}$ or $24 \mathrm{~h}$, neurological deficit scores assessed by measuring the focal deficits at $24 \mathrm{~h}$ after reperfusion were not significantly decreased (Fig. $3 C, P>0.05$ ). These results indicated that iAr application during ischemia over a duration range of $1-3 \mathrm{~h}$ provided the most efficacious neuroprotection and that prolonged inhalation for $24 \mathrm{~h}$ improved infarct volume.

\section{High concentrations alleviated brain damage better after ischemic stroke}

To further investigate whether the concentration of argon administration affected the neuroprotective effect of argon during ischemia on IMCAO-induced severe ischemic stroke in mice, we tested the neuroprotective effects of two different concentrations of argon (39\% argon- $40 \%$ nitrogen- $21 \%$ oxygen vs. $79 \%$ argon- $21 \%$ oxygen) initiated $1 \mathrm{~h}$ after stroke onset. The outcomes were brain infarct volume and neurological deficit scores. MCAO animals in the argon treatment groups exhibited reduced infarct volume compared with the $\mathrm{TMCAO}$ alone group regardless of the argon concentration (Fig. 4A and 4B; $\mathrm{P}<$ 0.001), and this was associated with improved neurological deficit scores assessed by measuring the focal deficits at $24 \mathrm{~h}$ after reperfusion (Fig. 4C; $\mathrm{P}<0.001$ ). In addition, we found that there was a significant difference between the 39\% iAr group and the 79\% iAr group in the neurologic outcome (neuroscore) but not in infarct volume. These results suggested that inhalation of $39 \%$ argon and $79 \%$ argon during ischemia both showed improved infarct volume and functional outcome $24 \mathrm{~h}$ after severe ischemic stroke, but the $79 \%$ argon inhalation group exhibited a better neurological outcome assessed by the general deficits compared with the $39 \%$ argon inhalation group.

\section{iAr promoted neurological recovery in both moderate and severe stroke models}

To explore whether iAr during ischemia could improve the outcomes of stroke of varying severity (moderate and severe ischemic stroke) induced by different ischemia durations, especially severe ischemic stroke induced by delayed reperfusion (not previously reported in vivo), and whether there are differences between the two ischemia durations in the argon treatment group, we used two different ischemia duration models (I/R $1.5 \mathrm{~h} / 24 \mathrm{~h} ; \mathrm{l} / \mathrm{R} 3 \mathrm{~h} / 24 \mathrm{~h}$ ) at the same treatment time point ( $1 \mathrm{~h}$ after stroke onset) with 3-hr argon therapeutic duration. Transient middle cerebral artery occlusion (tMCAO)-induced focal cerebral ischemia was performed as a $1.5 \mathrm{~h}(\mathrm{I} / \mathrm{R} 1.5 \mathrm{~h} / 24 \mathrm{~h})$ or $3 \mathrm{~h}(\mathrm{I} / \mathrm{R} 3 \mathrm{~h} / 24 \mathrm{~h})$ occlusion followed by $24 \mathrm{~h}$ reperfusion (Fig. 5A), which was assessed by the infarct volume, neurobehavioral scores, weight loss, and mortality rate $24 \mathrm{~h}$ after reperfusion (Fig. 5B-F). Delayed reperfusion induced a larger infarct volume in the MCAO mice compared to the $1.5 \mathrm{~h}$ occlusion and $24 \mathrm{~h}$ reperfusion model (Fig. 5B; $\mathrm{P}<$ 0.001). Moreover, MCAO mice with delayed reperfusion-induced stroke demonstrated more prominent neurological deficits on both the general and focal scales than the mice in the $1.5 \mathrm{~h}$ occlusion and $24 \mathrm{~h}$ reperfusion group (Fig. 5C, $\mathrm{P}<0.05 ; 5 \mathrm{D}, \mathrm{P}<0.001$ ). 
Similar to previous reports, we observed improved infarct volume, neurological deficit scores and weight recovery in the moderate ischemic stroke (I/R $1.5 \mathrm{~h} / 24 \mathrm{~h})$ group compared to the MCAO alone group (Fig. 5B-E). The results showed that iAr treatment in the severe stroke model led to significantly reduced infarct volume (Fig. 5B; $P<0.001$ ), improved neurological deficits on both the general and focal scales (Fig. 5C and 5D; $P<0.001$ ) and improved weight recovery (Fig. 5E; $P<0.001$ ). Of note, we found that compared with the iAr treatment group with severe ischemic stroke, the iAr treatment group with moderate ischemic stroke significantly improved infarct volume (Fig. 5B; $\mathrm{P}<0.001$ ) and neurological deficit scores assessed by the focal deficits (Fig. 5D; $\mathrm{P}<0.05$ ) at $24 \mathrm{~h}$ after reperfusion. However, weight recovery was improved by iAr to the same extent irrespective of the time of reperfusion. We also investigated the mortality rate at $24 \mathrm{~h}$ after MCAO reperfusion, and the results showed no significant benefit to the mortality rate in the iAr treatment group. Together, these results indicated that iAr treatment reduced infarct volume and improved neurological deficits and weight recovery after moderate and severe ischemic stroke induced by different ischemia durations, particularly in moderate stroke.

\section{Argon administration reduced edema after stroke onset}

We then investigated the effects of iAr on brain edema and neurologic impairment. MCAO/R mice with moderate ischemic stroke (I/R $1.5 \mathrm{~h} / 24 \mathrm{~h})$ showed a prominent increase in brain water content compared with those in the sham group (Fig. 6A; $\mathrm{P}<0.001$ ). Mice with moderate ischemic stroke treated with $\mathrm{iAr}$ showed significantly lower brain water content and neurological deficit scores (on both the general and focal scales) than those in the MCAO/R group (Fig. 6A-C; $P<0.001$ ). Similarly, MCAO/R mice with severe ischemic stroke demonstrated significantly higher brain water content and neurologic impairment. The argon treatment group showed significantly less brain edema and lower neurological deficit scores than the MCAO/R group (Fig. 6A-C; $\mathrm{P}<0.001$ ). The results showed that delayed reperfusion induced an increase in brain water content (Fig. 6A; $P<0.001$ ) and worse neurological deficits (Fig. 6B-C; $P<0.05)$ in the MCAO mice compared to the $1.5 \mathrm{~h}$ occlusion and $24 \mathrm{~h}$ reperfusion model. These results suggested that iAr treatment improved brain edema in moderate and severe ischemic stroke, especially moderate stroke.

\section{Discussion}

This study is the first to systematically explore the neuroprotective effect of iAr under different therapeutic schedules in IMCAO-induced moderate and severe ischemic stroke models. Our results suggest that compared to the IMCAO group, the groups administered $39 \%$ and $79 \%$ argon administration at $1 \mathrm{~h}$ after stroke onset for $1 \mathrm{~h}$ and $3 \mathrm{~h}$ showed a significant reduction in infarct volume in the severe cerebral ischemia model, and neurological outcome was obviously improved in a dose-dependent manner with $3 \mathrm{~h}$ of treatment. The neuroprotective efficacy of $79 \%$ argon administration at $1 \mathrm{~h}$ after stroke onset for $3 \mathrm{~h}$ was further validated in a moderate stroke model. Moreover, we found that the optimal schedule of argon treatment reduced edema in both models.

\section{The neuroprotective effects of inhaled argon on the infarct volume after ischemic stroke}


We observed that argon administration improved the infarct volume in severe ischemic stroke at $1 \mathrm{~h}$ after stroke onset (during ischemia) but not at $3 \mathrm{~h}$ after stroke onset (during reperfusion) in exp 1. Consistent with our observations during ischemia, Ryang et al[10] observed improved infarct volume when argon was administered for $1 \mathrm{~h}$ during ischemia after tMCAO induction. During reperfusion, David et al[11] found that argon decreased the subcortical volume but increased the subcortical volumes of damaged brain regions when given $1 \mathrm{~h}$ after reperfusion (during reperfusion), which is partly consistent with our results. Coburn et al[15] reported that the infarct volume was not affected by argon treatment with a $3 \mathrm{~h}$ delay after stroke onset and $1 \mathrm{~h}$ after reperfusion (during reperfusion). Similarly, Ma et al.[16] suggested that the infarct size was not decreased when argon was administered for $24 \mathrm{~h}$ during reperfusion. Notably, our results showed that argon administered for $1 \mathrm{~h}, 3 \mathrm{~h}$, or $24 \mathrm{~h}$ during ischemia decreased the infarct volume, especially at 1-3 h. We observed that during the trial by Ma et al[16], the initial application of argon for $24 \mathrm{~h}$ occurred during the reperfusion phase and, in our study, during the ischemic phase in exp 2. Overall, current evidence seems to show that iAr effectively improves the infarct volume when administered during ischemia, not reperfusion. The reasons for these results are unclear. One possible reason is that application of iAr immediately after reperfusion ( $3 \mathrm{~h}$ after ischemia) may miss the therapeutic window for neuroprotective intervention in the ischemic penumbra. Many animal and human experimental studies suggest that the therapeutic window for neuroprotective intervention in the ischemic penumbra is very brief, often less than 2 or $3 \mathrm{~h}[36,37]$. Another possible reason is that with the increase in the duration of ischemia, the ischemic core area is further enlarged, and ischemia-reperfusion injury may simply counteract or even exceed the protective effect of argon, as first proposed by Ma et al. Necroptosis and apoptosis are of great importance in cerebral ischemia-reperfusion injury, and the activation of necroptosis depends on reperfusion and is activated immediately after ischemic insult on reperfusion[38]. Reperfusion itself followed by MCAO can lead to neuronal death due to overperfusion and hemorrhagic transformation, which aggravates ischemic brain injury[39, 40]. Zhuang et al.[12] showed the effect of argon on the antiapoptotic signaling pathway in a neonatal rat asphyxia model. Therefore, we speculate that the antiapoptotic activity of argon may be offset or even concealed by this reperfusion injury to some extent. Moreover, recent literature[15] has reported that treatment with argon promotes the switch of microglia/macrophage polarization toward the anti-inflammatory M2 phenotype. In addition, cerebral ischemia and ischemia coupled with reperfusion result in differing pathologic mechanisms and microglial morphological responses, and a spatiotemporal relationship exists between microglial morphology and evolving brain injury after ischemic stroke and reperfusion[41]. We speculate that iAr can impact the balance between proinflammatory and anti-inflammatory cytokines to antiinflammation after AIS and affects the infarct size under the appropriate combination of treatment timing and duration of treatment (for example, short-term intervention during ischemia). However, this is just our speculation, and additional experiments are needed to validate this hypothesis and further dissect the possible mechanisms involved.

\section{Recovery of neurological function with inhaled argon after ischemic stroke}

Functional recovery is one of the main end outcomes of stroke patients. Clinically, it is customary to divide the neurological impairment of patients with stroke into general functional injury and focal 
functional injury. The former reflects the patient's life state and global neurological function, while the latter reflects localized functional defects caused by local injury. Therefore, in addition to infarct volume, neurological deficits were assessed in this study according to Clark's scoring system[33], reflecting the degree of neurological injury after ischemia, from the point of view of general functional injury and focal functional injury. Our in vivo results suggested that argon administered at $39 \mathrm{vol} \%$ and $79 \mathrm{vol} \%$ could improve neurologic outcome in exp 3 in a dose-dependent manner. In accordance with our results, previous studies suggest that argon exerts neuroprotective effects in a dose-dependent manner in different injury models (hypoxia vs. ischemia), such as models of traumatic brain injury (TBI)[9], retinal ischemia/reperfusion injury (IRI)[42] and cardiac arrest (CA)[43]. Similarly, Coburn et al[15]. demonstrated significantly improved neurological performance using a 6-point neuroscore daily from $24 \mathrm{~h}$ to $7 \mathrm{~d}$ after reperfusion. In addition, Ma et al.[16] found that neurologic outcome was significantly improved after 24 $\mathrm{h}$ of treatment with argon instituted after reperfusion. However, in contrast with its beneficial effect on the neurological outcome, David et al.[11] found that $50 \mathrm{vol} \%$ argon inhaled during reperfusion for $3 \mathrm{~h}$ failed to improve MCAO-induced neurologic deficits. The results seem to indicate that even if argon is administered at similar time points (such as after reperfusion), it will lead to significant differences in neurological impairment due to different treatment protocols, such as durations and concentrations. Notably, however, in addition to the initial time point of iAr, the difference in neurologic assessment systems is one factor to be considered.

An interesting aspect of our research is that argon administration for $1 \mathrm{~h}$ or $24 \mathrm{~h}$ during ischemia reduced infarct volume, but neurologic outcomes were not improved. Another unanticipated result was that iAr caused dose-dependent improvement of neurological prognosis in our study, which was also inconsistent with observations of infarct volume. These results reflect those of Coburn et al.[15], who found that with a $3 \mathrm{~h}$ delay after stroke onset and $1 \mathrm{~h}$ after reperfusion, argon significantly alleviated neurological deficits during the first week after stroke but failed to reduce the infarct volume. Additionally, these differences corroborate the ideas of Ma et al.[16], who found that neurologic outcome was improved but that infarct size was not reduced when argon administration was delayed $2 \mathrm{~h}$ after permanent stroke onset or instituted after reperfusion. One possible explanation of this difference may be that although there is a correlation between the focal functional injury score and cerebral infarction volume of the Clark score, evidence from the recent literature indicates that histologic lesion size was less correlated with improved neurologic outcome, as $\mathrm{Ma}$ and colleagues clearly pointed out.

\section{The influences of argon on edema after ischemic stroke}

Few studies have addressed the effect of iAr on the complications of AIS. However, the complications of AIS have significant effects on the short-term and long-term prognosis of patients, and brain edema is a common complication of AIS and one of the main causes of death. Acute brain injury is linked to cellular edema, characterized by abnormal intracellular accumulation of water in brain cells, resulting in cellular swelling[44]. Delayed reperfusion, whether by thrombolysis or endovascular therapy, can result in more severe brain edema and poorer clinical outcomes and increase the risk of mortality, hemorrhagic transformation, headache, and seizures[45, 46]. Brain edema is also an important prognostic indicator in 
the assessment of argon efficacy in severe ischemic stroke induced by delayed reperfusion[40]. As previously reported[10,11, 15, 47,48], argon has been shown to exert a neuroprotective effect in in vivo tMCAO models induced by ischemia for $1-2 \mathrm{~h}$, consistent with our findings. Our study showed that neuroprotection still existed in severe ischemic stroke induced by an increasing ischemic duration beyond those commonly used at clinically relevant time windows. The results showed that argon treatment in moderate and severe AIS models led to significantly reduced brain water content, infarct volume, neurologic function, and weight recovery at the same treatment time point, duration and concentration. Importantly, delayed reperfusion affects the ability of iAr to improve brain edema, infarction volume and neurologic function following IMCAO. The design of the present study allowed conclusions to be drawn regarding whether a higher concentration of argon inhaled for $3 \mathrm{~h}$ during ischemia would improve the complications characterized by brain edema in TMCAO model. Further studies are needed to clarify the underlying mechanism of this improvement and should pay more attention to argon-induced improvements of other complications of AIS, such as hemorrhagic transformation.

\section{Limitations and prospective}

There are several design limitations in our experiment that deserve special attention. First, an important limitation is the lack of data to measure potential medium- and long-term effects, which are clinically important for the prognosis of patients with acute ischemic stroke. Animals survived for only $24 \mathrm{~h}$ after reperfusion, which may contribute to some results that are not wholly consistent with the conclusions of our study at a later sampling time. Longer recovery times should be included in future studies, and functional imaging, such as MRI, can be applied to monitor the evolution of brain injury and treatment. Second, animal models other than rodents should be adopted, including large animal models[49, 50], such as pigs, dogs and monkeys. Further research should test the same strategies in larger species with basic diseases such as hypertension or diabetes and explore the impact of other biological variables such as sex and age. These factors were not explored in this study because of budgetary limitations. Finally, this study is limited because it considered only the timing, duration and concentration; however, the specific mechanism involved in this improvement was not investigated, and this is part of our future research contents and direction. Thus, further animal and clinical studies addressing these issues at length are needed. Despite these limitations, this study is the first to demonstrate the effects of argon on cellular edema after MCAO-induced severe cerebral ischemic stroke in mice. Moreover, our study adopted the synthesis of two kinds of neurobehavioral scores that are suitable for clinical practice, and the behavioral experiments were double blinded to limit bias.

\section{Conclusions}

In this study, it was demonstrated that timely iAr administration during ischemia for appropriate shortterm treatment at a higher concentration provides better neuroprotective efficacy. We conceived this study as an instructive guide for the protective effects of argon on AIS and better clinical translation.

\section{Abbreviations}


$\mathrm{i} A r=$ inhaled argon; AIS = acute ischemic stroke; I/R = ischemic/reperfusion; IRI = ischemia/reperfusion injury; $\mathrm{tMCAO}=$ transient middle cerebral artery occlusion; $\mathrm{MCA}=$ middle cerebral artery; $\mathrm{Ar}=$ argon; $\mathrm{H}_{2} \mathrm{~S}=$ hydrogen sulfide; $\mathrm{H}_{2}$ = hydrogen; $\mathrm{BBB}$ = blood-brain barrier; $\mathrm{HIE}$ = hypoxic-ischemic encephalopathy; $\mathrm{TBI}$ $=$ traumatic brain injury; $\mathrm{CA}=$ cardiac arrest; $\mathrm{TGF} \beta=$ transforming growth factor $-\beta ; \mathrm{NGF}=$ nerve growth factor; VEGF = vascular endothelial growth factor; IL-1 $\beta=$ interleukin-1 $\beta$; IL- $6=$ interleukin- 6 ; iNOS = induced-type nitric oxide synthase; $\mathrm{CCA}=$ common carotid artery; ECA= external carotid artery; ICA= internal carotid artery; TTC = 2,3,5-tripenyltetrazolium chloride; $\mathrm{ANOVA}=$ analysis of variance; $\mathrm{MRI}=$ Magnetic Resonance Imaging.

\section{Declarations}

\section{Ethics approval and consent to participate}

The research protocol and animal care procedures of this study were inspected and approved by the Laboratory Animal Center and Lab Animal Ethical Committee of Nantong University.

\section{Consent for publication}

Not applicable.

\section{Availability of data and materials}

The datasets used and analyzed during the current study are available from the corresponding author on reasonable request.

\section{Competing interests}

The authors declare that they have no competing interests.

\section{Funding}

This study was supported by Postgraduate Research \& Practice Innovation Program of Jiangsu Province (no. SJCX20_1168).

\section{Authors' contributions}

YFZ and XL conceived and designed the study, supervised the work, analyzed and interpreted data, and wrote the manuscript, revised, and final approved. JH was involved in experimental conception, first draft of manuscript, analysis and interpretation of data. KX was involved in acquisition of data, analysis and interpretation of data. JYL helped with TMCAO model and double-blind analysis. JHG was involved in interpretation of data. BP and LHX helped with gas ventilation and animal supply according to the experimental protocol. GHW and ZLJ helped to design and evaluate the study. All authors read and approved the final manuscript. 
Acknowledgements

We thank Zhihua Gao for language editing.

\section{References}

1. Global, regional, and national age-sex specific mortality for 264 causes of death, 1980-2016: a systematic analysis for the Global Burden of Disease Study 2016. Lancet (London, England) 2017, 390(10100):1151-1210.

2. Robba C, Bonatti G, Battaglini D, Rocco PRM, Pelosi P: Mechanical ventilation in patients with acute ischaemic stroke: from pathophysiology to clinical practice. Critical Care 2019, 23(1).

3. Moretti A, Ferrari F, Villa RF: Neuroprotection for ischaemic stroke: current status and challenges. Pharmacol Ther 2015, 146:23-34.

4. Deng J, Lei C, Chen Y, Fang Z, Yang Q, Zhang H, Cai M, Shi L, Dong H, Xiong L: Neuroprotective gases-fantasy or reality for clinical use? Prog Neurobiol 2014, 115:210-245.

5. Fahlenkamp AV, Rossaint R, Coburn M: [Neuroprotection by noble gases: New developments and insights]. Anaesthesist 2015, 64(11):855-858.

6. Dickinson R, Franks N: Bench-to-bedside review: Molecular pharmacology and clinical use of inert gases in anesthesia and neuroprotection. Critical care (London, England) 2010, 14(4):229.

7. Winkler DA, Thornton A, Farjot G, Katz I: The diverse biological properties of the chemically inert noble gases. Pharmacol Ther 2016, 160:44-64.

8. Jawad N, Rizvi M, Gu J, Adeyi O, Tao G, Maze M, Ma D: Neuroprotection (and lack of neuroprotection) afforded by a series of noble gases in an in vitro model of neuronal injury. Neurosci Lett 2009, 460(3):232-236.

9. Loetscher PD, Rossaint J, Rossaint R, Weis J, Fries M, Fahlenkamp A, Ryang YM, Grottke O, Coburn M: Argon: neuroprotection in in vitro models of cerebral ischemia and traumatic brain injury. Crit Care 2009, 13(6):R206.

10. Ryang YM, Fahlenkamp AV, Rossaint R, Wesp D, Loetscher PD, Beyer C, Coburn M: Neuroprotective effects of argon in an in vivo model of transient middle cerebral artery occlusion in rats. Crit Care Med 2011, 39(6):1448-1453.

11. David HN, Haelewyn B, Degoulet M, Colomb DG, Jr., Risso JJ, Abraini JH: Ex vivo and in vivo neuroprotection induced by argon when given after an excitotoxic or ischemic insult. PLoS One 2012, 7(2):e30934.

12. Zhuang L, Yang T, Zhao H, Fidalgo AR, Vizcaychipi MP, Sanders RD, Yu B, Takata M, Johnson MR, Ma D: The protective profile of argon, helium, and xenon in a model of neonatal asphyxia in rats. Crit Care Med 2012, 40(6):1724-1730.

13. Broad KD, Fierens I, Fleiss B, Rocha-Ferreira E, Ezzati M, Hassell J, Alonso-Alconada D, Bainbridge A, Kawano G, Ma D et al: Inhaled 45-50\% argon augments hypothermic brain protection in a piglet 
model of perinatal asphyxia. Neurobiology of Disease 2016, 87:29-38.

14. Grusser L, Blaumeiser-Debarry R, Krings M, Kremer B, Hollig A, Rossaint R, Coburn M: Argon attenuates the emergence of secondary injury after traumatic brain injury within a 2-hour incubation period compared to desflurane: an in vitro study. Med Gas Res 2017, 7(2):93-100.

15. Liu J, Nolte K, Brook G, Liebenstund L, Weinandy A, Hollig A, Veldeman M, Willuweit A, Langen KJ, Rossaint $\mathrm{R}$ et al: Post-stroke treatment with argon attenuated brain injury, reduced brain inflammation and enhanced M2 microglia/macrophage polarization: a randomized controlled animal study. Crit Care 2019, 23(1):198.

16. Ma S, Chu D, Li L, Creed JA, Ryang YM, Sheng H, Yang W, Warner DS, Turner DA, Hoffmann U: Argon Inhalation for 24 Hours After Onset of Permanent Focal Cerebral Ischemia in Rats Provides Neuroprotection and Improves Neurologic Outcome. Crit Care Med 2019, 47(8):e693-e699.

17. Moro F, Fossi F, Magliocca A, Pascente R, Sammali E, Baldini F, Tolomeo D, Micotti E, Citerio G, Stocchetti $\mathrm{N}$ et al: Efficacy of acute administration of inhaled argon on traumatic brain injury in mice. Br J Anaesth 2020.

18. Moro F, Fossi F, Magliocca A, Pascente R, Sammali E, Baldini F, Tolomeo D, Micotti E, Citerio G, Stocchetti $\mathrm{N}$ et al: Efficacy of acute administration of inhaled argon on traumatic brain injury in mice. British Journal of Anaesthesia 2021, 126(1):256-264.

19. Edge CJ, Dickinson R: Argon: a noble, but not inert, treatment for brain trauma? Br J Anaesth 2021, 126(1):41-43.

20. Hollig A, Weinandy A, Liu J, Clusmann H, Rossaint R, Coburn M: Beneficial Properties of Argon After Experimental Subarachnoid Hemorrhage: Early Treatment Reduces Mortality and Influences Hippocampal Protein Expression. Crit Care Med 2016, 44(7):e520-529.

21. Kremer B, Coburn M, Weinandy A, Nolte K, Clusmann H, Veldeman M, Hollig A: Argon treatment after experimental subarachnoid hemorrhage: evaluation of microglial activation and neuronal survival as a subanalysis of a randomized controlled animal trial. Med Gas Res 2020, 10(3):103-109.

22. Ulbrich F, Lerach T, Biermann J, Kaufmann KB, Lagreze WA, Buerkle H, Loop T, Goebel U: Argon mediates protection by interleukin-8 suppression via a TLR2/TLR4/STAT3/NF-kappaB pathway in a model of apoptosis in neuroblastoma cells in vitro and following ischemia-reperfusion injury in rat retina in vivo. J Neurochem 2016, 138(6):859-873.

23. Zhao H, Mitchell S, Koumpa S, Cui Y, Lian Q, Hagberg H, Johnson M, Takata M, Ma D: Heme Oxygenase-1 Mediates Neuroprotection Conferred by Argon in Combination with Hypothermia in Neonatal Hypoxia-Ischemia Brain Injury. Anesthesiology 2016, 125(1):180-192.

24. Sanders RD, Ma D, Maze M: Argon neuroprotection. Crit Care 2010, 14(1):117.

25. Ezzeddine MA: From xenon to argon: a more clinically accessible neuroprotectant? Crit Care Med 2011, 39(6):1589-1590.

26. Coburn M, Rossaint R: Argon in the fast lane: noble gases and their neuroprotective effects. Crit Care Med 2012, 40(6):1965-1966. 
27. David HN, Haelewyn B, Risso J-J, Abraini JH: Modulation by the noble gas argon of the catalytic and thrombolytic efficiency of tissue plasminogen activator. Naunyn-Schmiedeberg's Archives of Pharmacology 2012, 386(1):91-95.

28. Gardner AJ, Menon DK: Moving to human trials for argon neuroprotection in neurological injury: a narrative review. Br J Anaesth 2018, 120(3):453-468.

29. Koziakova M, Harris K, Edge CJ, Franks NP, White IL, Dickinson R: Noble gas neuroprotection: xenon and argon protect against hypoxic-ischaemic injury in rat hippocampus in vitro via distinct mechanisms. Br J Anaesth 2019, 123(5):601-609.

30. Creed J, Cantillana-Riquelme V, Yan BH, Ma S, Chu D, Wang H, Turner DA, Laskowitz DT, Hoffmann U: Argon Inhalation for $24 \mathrm{~h}$ After Closed-Head Injury Does not Improve Recovery, Neuroinflammation, or Neurologic Outcome in Mice. Neurocrit Care 2021, 34(3):833-843.

31. Fahlenkamp A, Coburn M, de Prada A, Gereitzig N, Beyer C, Haase H, Rossaint R, Gempt J, Ryang Y: Expression analysis following argon treatment in an in vivo model of transient middle cerebral artery occlusion in rats. Medical gas research 2014, 4:11.

32. Xu X, Zhu L, Xue K, Liu J, Wang J, Wang G, Gu Jh, Zhang Y, Li X: Ultrastructural studies of the neurovascular unit reveal enhanced endothelial transcytosis in hyperglycemia-enhanced hemorrhagic transformation after stroke. CNS Neuroscience \& Therapeutics 2021, 27(1):123-133.

33. Clark W, Lessov N, Dixon M, Eckenstein F: Monofilament intraluminal middle cerebral artery occlusion in the mouse. Neurological research 1997, 19(6):641-648.

34. Swanson R, Morton M, Tsao-Wu G, Savalos R, Davidson C, Sharp F: A semiautomated method for measuring brain infarct volume. Journal of cerebral blood flow and metabolism : official journal of the International Society of Cerebral Blood Flow and Metabolism 1990, 10(2):290-293.

35. Hu X, Li S, Doycheva DM, Huang L, Lenahan C, Liu R, Huang J, Xie S, Tang J, Zuo G et al: Rh-CSF1 attenuates neuroinflammation via the CSF1R/PLCG2/PKCepsilon pathway in a rat model of neonatal HIE. J Neuroinflammation 2020, 17(1):182.

36. Heiss W, Thiel A, Grond M, Graf R: Which targets are relevant for therapy of acute ischemic stroke? Stroke 1999, 30(7):1486-1489.

37. Lees K: Neuroprotection is unlikely to be effective in humans using current trial designs: an opposing view. Stroke 2002, 33(1):308-309.

38. Naito MG, Xu D, Amin P, Lee J, Wang H, Li W, Kelliher M, Pasparakis M, Yuan J: Sequential activation of necroptosis and apoptosis cooperates to mediate vascular and neural pathology in stroke. Proc Natl Acad Sci U S A 2020, 117(9):4959-4970.

39. Guan X, Zhang H, Qin H, Chen C, Hu Z, Tan J, Zeng L: CRISPR/Cas9-mediated whole genomic wide knockout screening identifies mitochondrial ribosomal proteins involving in oxygen-glucose deprivation/reperfusion resistance. Journal of cellular and molecular medicine 2020, 24(16):93139322.

40. Tang C, Hu Y, Lyu H, Gao J, Jiang J, Qin X, Wu Y, Wang J, Chai X: Neuroprotective effects of 1-O-hexyl2,3,5-trimethylhydroquinone on ischaemia/reperfusion-induced neuronal injury by activating the 
Nrf2/H0-1 pathway. Journal of cellular and molecular medicine 2020, 24(18):10468-10477.

41. Morrison $\mathrm{H}$, Filosa J: A quantitative spatiotemporal analysis of microglia morphology during ischemic stroke and reperfusion. Journal of neuroinflammation 2013, 10:4.

42. Das A, Ulbrich F, Schallner N, Coburn M, Loop T, Lagrèze WA, Biermann J, Goebel U: Argon Inhalation Attenuates Retinal Apoptosis after Ischemia/Reperfusion Injury in a Time-and Dose-Dependent Manner in Rats. PLOS ONE 2014, 9(12).

43. Brucken A, Kurnaz P, Bleilevens C, Derwall M, Weis J, Nolte K, Rossaint R, Fries M: Dose dependent neuroprotection of the noble gas argon after cardiac arrest in rats is not mediated by K(ATP)-channel opening. Resuscitation 2014, 85(6):826-832.

44. Kishimoto M, Suenaga J, Takase H, Araki K, Yao T, Fujimura T, Murayama K, Okumura K, Ueno R, Shimizu $\mathrm{N}$ et al: Oxidative stress-responsive apoptosis inducing protein (ORAIP) plays a critical role in cerebral ischemia/reperfusion injury. Sci Rep 2019, 9(1):13512.

45. Churchill NW, Hutchison MG, Richards D, Leung G, Graham SJ, Schweizer TA: Neuroimaging of sport concussion: persistent alterations in brain structure and function at medical clearance. Sci Rep 2017, 7(1):8297.

46. Zhao W, Wu C, Dornbos D, Li S, Song H, Wang Y, Ding Y, Ji X: Multiphase adjuvant neuroprotection: A novel paradigm for improving acute ischemic stroke outcomes. Brain circulation 2020, 6(1):11-18.

47. Hollig A, Schug A, Fahlenkamp AV, Rossaint R, Coburn M, Argon Organo-Protective N: Argon: systematic review on neuro- and organoprotective properties of an "inert" gas. Int J Mol Sci 2014, 15(10):18175-18196.

48. De Deken J, Rex S, Monbaliu D, Pirenne J, Jochmans I: The Efficacy of Noble Gases in the Attenuation of Ischemia Reperfusion Injury. Critical Care Medicine 2016, 44(9):e886-e896.

49. Humphray S, Scott C, Clark R, Marron B, Bender C, Camm N, Davis J, Jenks A, Noon A, Patel M et al: A high utility integrated map of the pig genome. Genome biology 2007, 8(7):R139.

50. Zhang Y, Jin M, Du B, Lin H, Xu C, Jiang W, Jia J: A Novel Canine Model of Acute Vertebral Artery Occlusion. PloS one 2015, 10(11):e0142251.

\section{Figures}



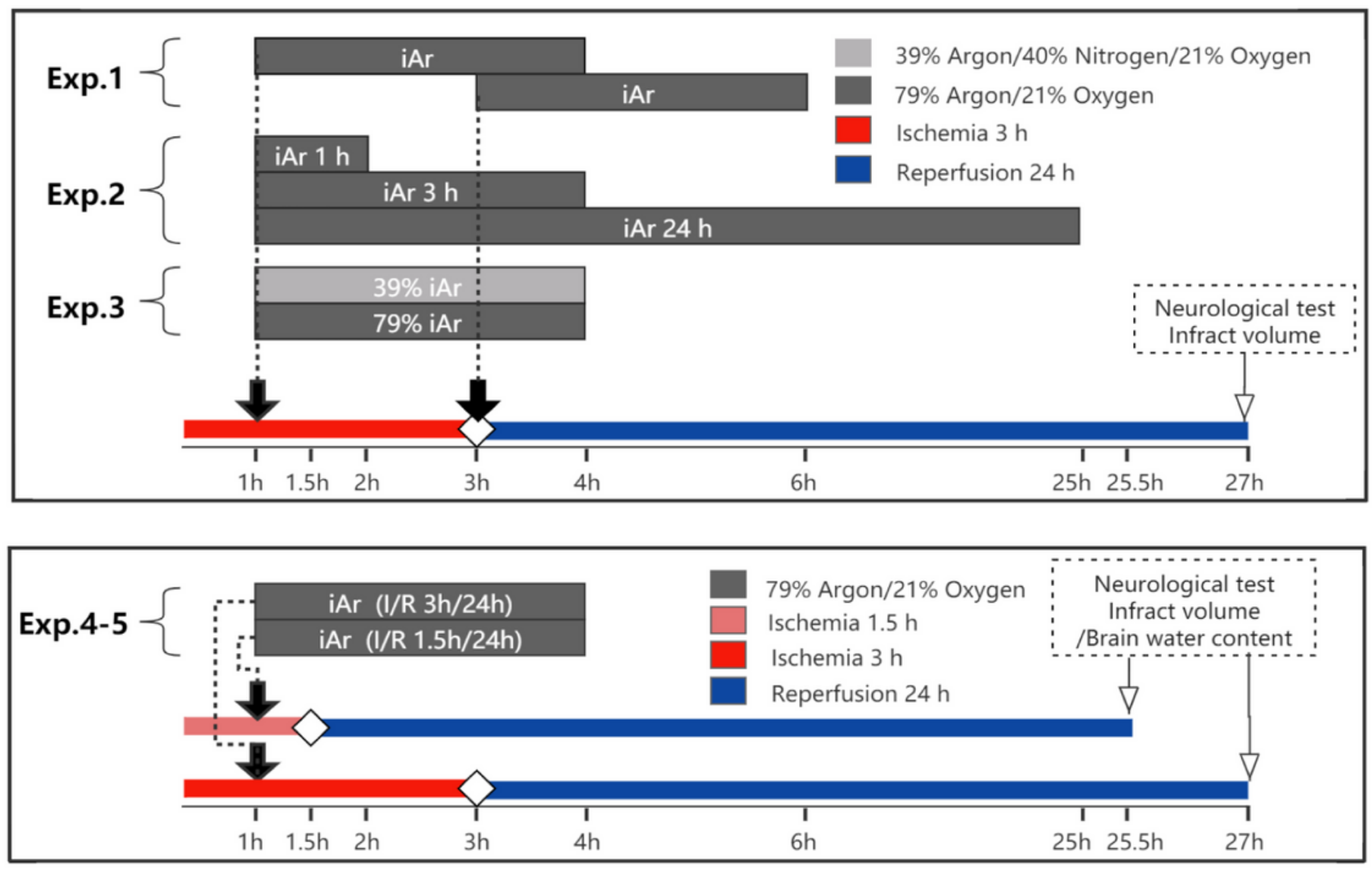

\section{Figure 1}

Schematic illustration of the experimental design of inhaled argon (iAr). The red box represents the period of ischemia. The blue box represents the period of reperfusion. 39\% iAr, inhaled 39\% argon- $40 \%$ nitrogen$21 \%$ oxygen; $79 \%$ iAr, inhaled $79 \%$ argon- $21 \%$ oxygen; A concentration of $79 \%$ iAr was used for all experiments except the dose-response experiment 3 , where doses are indicated. 
A
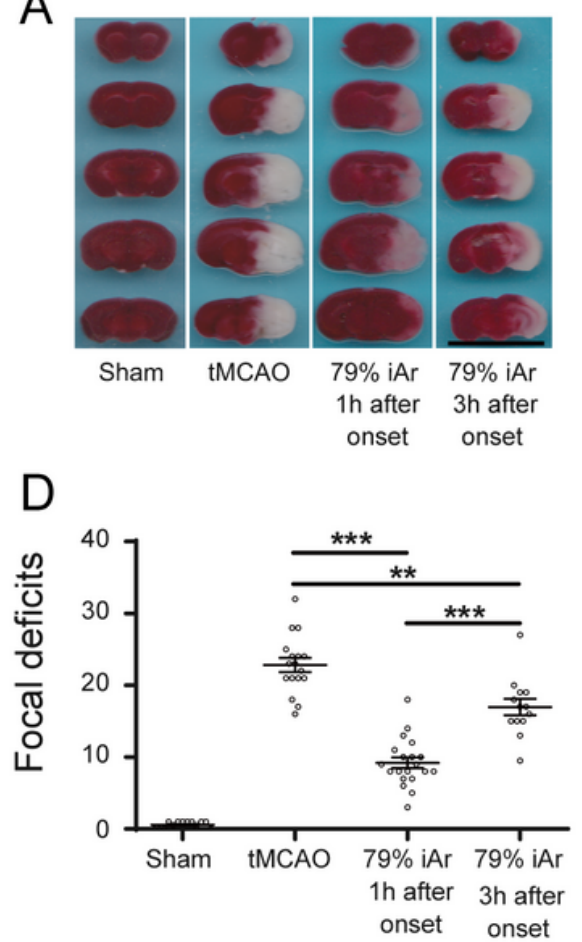
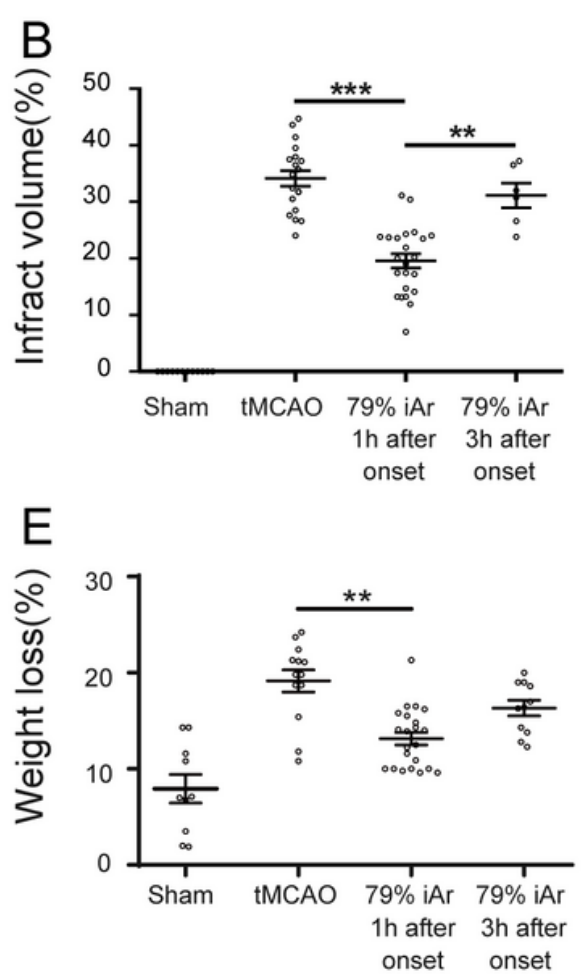
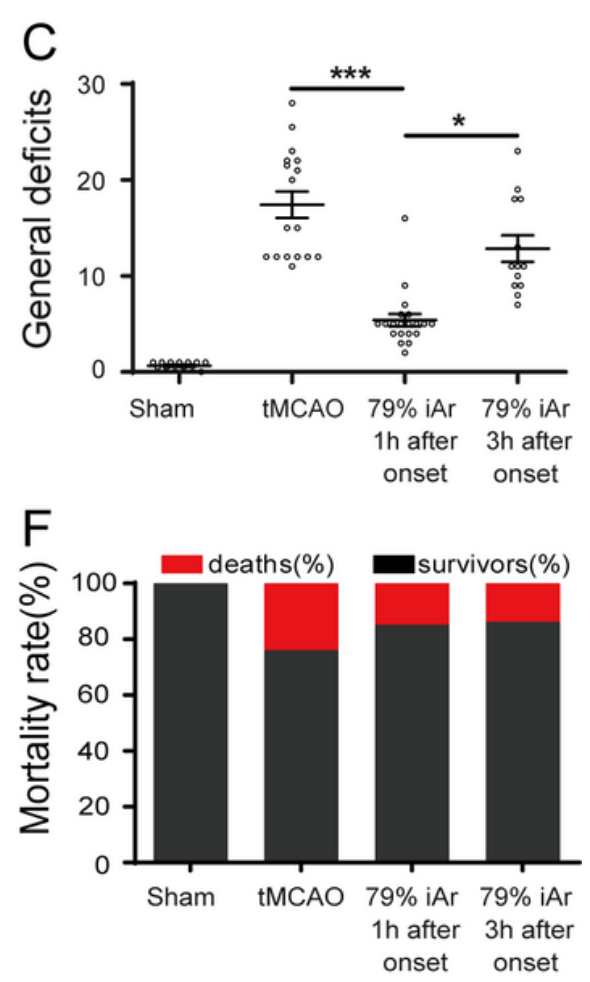

\section{Figure 2}

The neuroprotective effects of $\mathrm{iAr}(79 \%)$ application $1 \mathrm{~h}$ and $3 \mathrm{~h}$ after stroke onset. A, Representative 2,3,5-triphenyltetrazolium chloride (TTC) staining was performed to assess the infarct volume of mice at $24 \mathrm{~h}$ after reperfusion in the four groups(scale bar: $10 \mathrm{~mm}$ ). B, Edema-corrected infarct volume after tMCAO with reperfusion, $3 \mathrm{~h}$ iAr administration, and $1 \mathrm{~d}$ recovery ( $\mathrm{n}=6$ to $23 /$ group). Reduced stroke volumes after iAr administration $1 \mathrm{~h}$ after stroke onset also translated into a better functional outcome at $24 \mathrm{~h}$ as assessed by the general deficits ( $\mathrm{n}=12$ to 20/group; $C$, and the focal deficits ( $n=12$ to 20/group; $D$ and $\mathrm{E}$, overall recovery based on weight at baseline before stroke and after $3 \mathrm{~h}$ iAr administration and 24 $\mathrm{h}$ recovery ( $\mathrm{n}=10$ to $22 /$ group;). F. Mortality after $3 \mathrm{~h}$ of transient middle cerebral artery occlusion and $24 \mathrm{~h}$ postreperfusion. Deaths occurred within $24 \mathrm{~h}$ after ischemia/reperfusion. Death (marked red) occurred within the first $24 \mathrm{~h}$. The results are represented as the mean \pm SEM. ${ }^{*} P<0.05,{ }^{\star} * P<0.01,{ }^{\star} * \star P<0.001$. Statistical comparisons were carried out with one-way ANOVA.

A

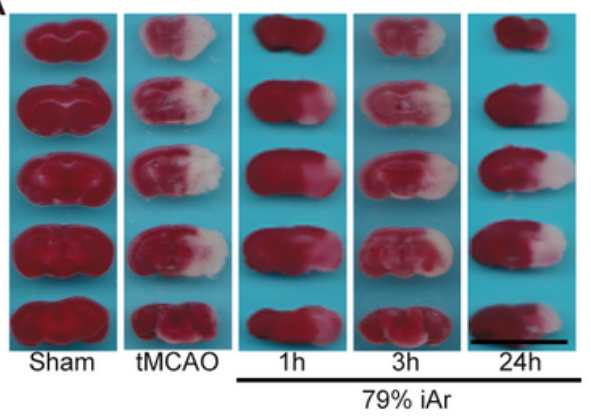

B

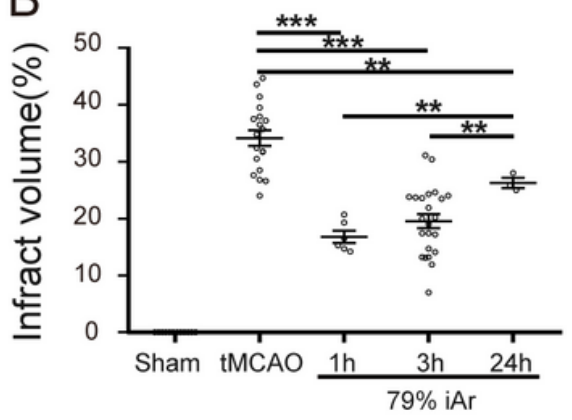

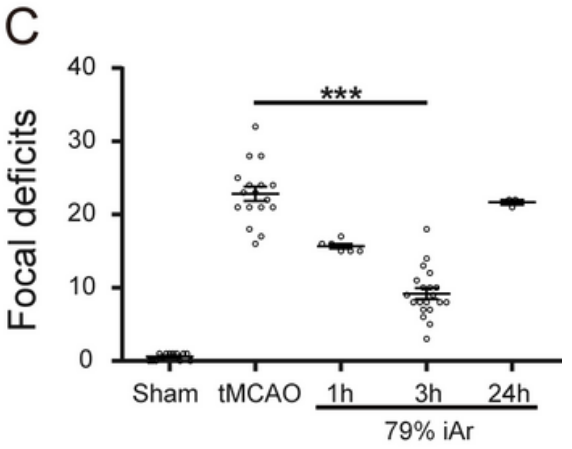

Figure 3 
Duration of $\mathrm{iAr}(79 \%)$ application for neuroprotective effects. A, Representative coronal brain slices stained with TTC (scale bar: $10 \mathrm{~mm}$ ). Infarct volumes ( $\mathrm{n}=3$ to 23/group; B) and functional outcomes ( $\mathrm{n}=3$ to 23/group; C) on day 1 after $3 \mathrm{~h}$ of transient middle cerebral artery occlusion in mice with three different durations of iAr application (inhaled $1 \mathrm{~h}$ after stroke onset). The results were represented as the mean \pm SEM. ${ }^{*} P<0.05,{ }^{*} P<0.01,{ }^{*} * * P<0.001$. Statistical comparisons were carried out with one-way ANOVA.
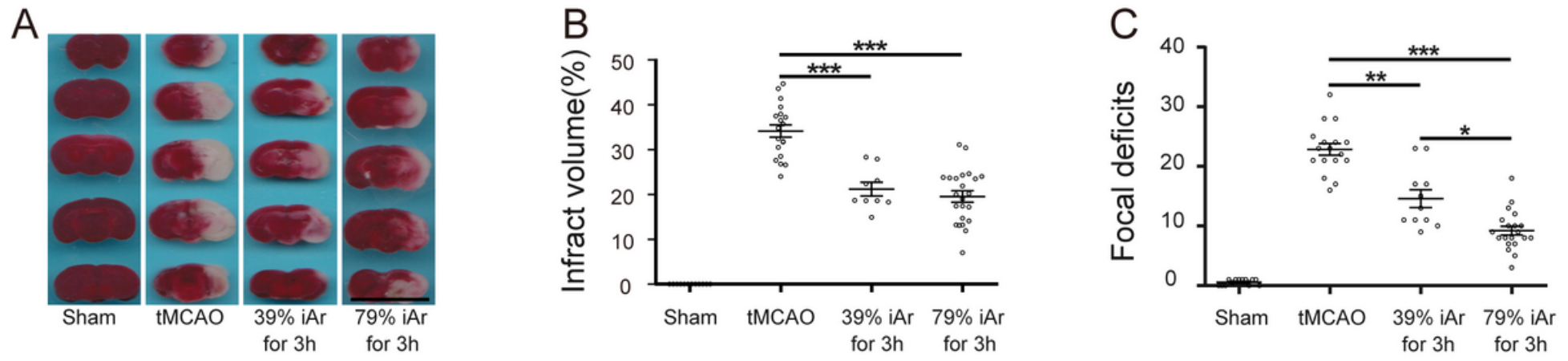

Figure 4

39\% iAr and 79\% iAr application for a neuroprotective effect. A, Representative coronal brain slices stained with TTC (scale bar: $10 \mathrm{~mm}$ ). Infarct volumes ( $\mathrm{n}=9$ to 23/group; B) and functional outcomes ( $n=11$ to 23 /group; $C$ ) on day 1 after $3 \mathrm{~h}$ of transient middle cerebral artery occlusion in mice treated with two different doses of argon (argon administration $1 \mathrm{~h}$ after stroke onset). The results are represented as the mean \pm SEM. ${ }^{*} P<0.05,{ }^{*} P<0.01,{ }^{\star} * * P<0.001$. Statistical comparisons were carried out with one-way ANOVA. 39\% iAr, inhaled 39\% argon-40\% nitrogen-21\% oxygen; 79\% iAr, inhaled 79\% argon-21\% oxygen.
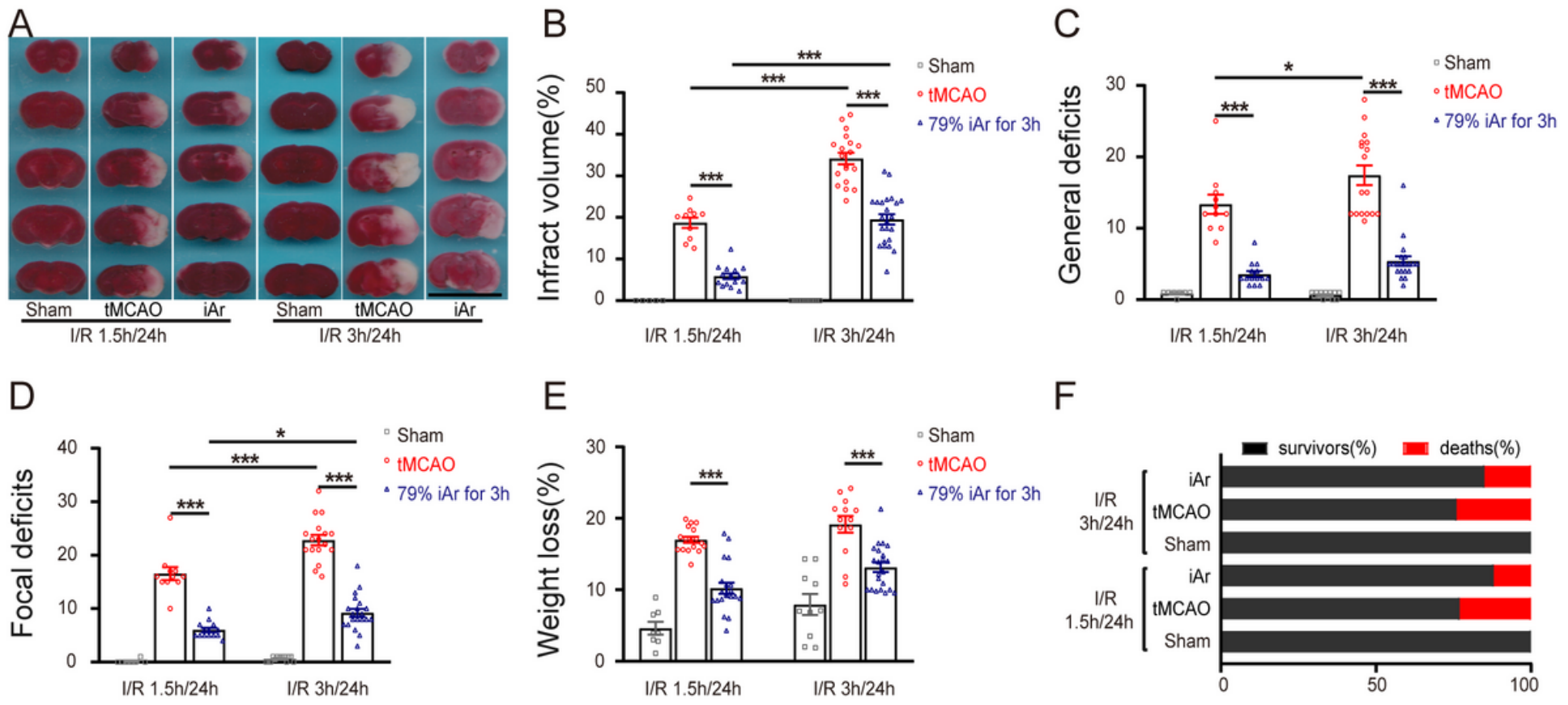

\section{Figure 5}

In two models of ischemic duration, iAr (79\%) was applied for a neuroprotective effect. A, Representative images of TTC-stained cerebral coronal sections of mice(scale bar: $10 \mathrm{~mm}$ ) in the sham, tMCAO, and 
argon treatment groups in two different time windows between ischemia and reperfusion (I/R $1.5 \mathrm{~h} / 24 \mathrm{~h}$; I/R 3 h/24 h). Quantification of brain infarct volume (B, $n=5$ to 23/group), neurobehavioral scores (C-D, $n=7$ to 20/group) and weight loss ( $E, n=8$ to 22/group) $24 \mathrm{~h}$ after reperfusion in tMCAO- and argon-treated mice. $\mathrm{F}$, The mortality rate $24 \mathrm{~h}$ after ischemia/reperfusion in two different durations of ischemia. Data are shown as the mean \pm SEM. ${ }^{*} P<0.05,{ }^{*} P<0.01, * \star * P<0.001$. Statistical comparisons were carried out with two-way ANOVA.

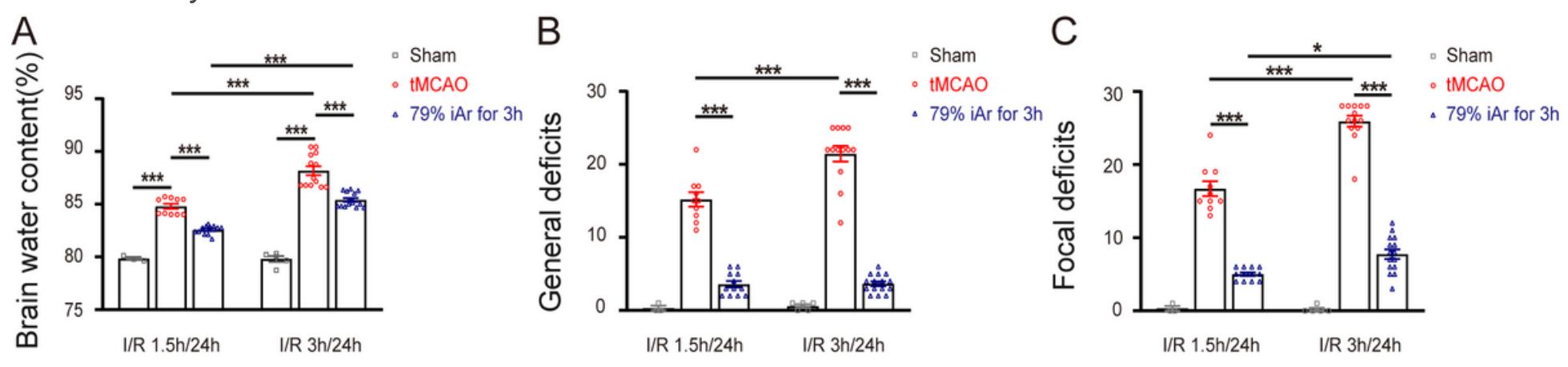

\section{Figure 6}

Brain edema, measured by the percentage of brain water content of each part $24 \mathrm{~h}$ after ischemia/reperfusion in the three groups ( $n=3$ to 15/group). Quantification of neurobehavioral scores 24 $h$ after reperfusion as assessed by general deficits ( $n=3$ to $15 /$ group; $C$ ) and focal deficits $(n=3$ to 15 /group; D). Data are shown as the mean \pm SEM. ${ }^{*} P<0.05$, ${ }^{\star *} P<0.01,{ }^{\star} * * P<0.001$. Statistical comparisons were carried out with two-way ANOVA. 